\title{
El caleidoscopio urbano en el mundo "céltico" de la Meseta
}

\author{
The urban caleidoscope in the Meseta "celtic" world
}

\author{
Gonzalo Ruiz ZaPATERo \\ Departamento de Prehistoria. Universidad Complutense, 28040-Madrid \\ gonzalor@ghis.ucm.es
}

Recibido: 17-01-2011

Aceptado: 10-05-2011

\section{RESUMEN}

A lo largo del primer milenio a.C. se desarrollan en la Meseta Norte española un proceso de crecimiento demográfico y una tendencia al hábitat nucleado que acabarán desembocando en las grandes ciudades celtíberas, vacceas y vettonas, conocidas por la arqueología y, algunas al menos, citadas por las fuentes clásicas. Pero el proceso dista de ser homogéneo, más bien fue en mosaico según las distintas regiones de la Meseta Norte y, además, no adoptó una evolución lineal; de manera que los ritmos y formas de agrupación de las comunidades fueron diversos según las distintas regiones de la Meseta Norte. Al final la visión del proceso que condujo a la primera urbanización a finales de la Edad del Hierro se presenta como una realidad caleidoscópica, cambiante en el espacio y el tiempo, con similitudes y especificidades propias.

Palabras Clave: Edad del Hierro. Urbanismo. Aldeas. Ciudades. Oppida. Meseta Norte. Poblamiento.

\begin{abstract}
Throughout the first millennium BC two processes took place in the Spanish Northern Meseta: a process of increasing population and a trend towards nucleated settlements. Both processes led to the emergence of the big Celtiberian, Vaccean and Vetton cities, well known in the archaeological record and, at least some of them, by the written historical sources. The process was not lineal and homogeneous, but rather a mosaiclike one, with rhythms and settlement forms different for each region of the Northern Meseta. In the end, the scene shown by the developing process conducting to the first urbanization at the end of the Iron Age is presented in this paper as a kaleidoscopic entity, changing in space and time, with its affinities and own features.
\end{abstract}

KeY words: Iron Age. Urbanism. Villages. Cities. Oppida. North Meseta (Spain). Settlement.

Sumario: Introducción. 1. El proceso de urbanización en la Edad del Hierro de la Meseta. 2. Perspectivas de futuro y necesidades de la investigación. 
El concepto de urbano en la Edad del Hierro de la Europa Templada no es comparable al urbanismo mediterráneo, especialmente el griego e itálico, y antes de los oppida de finales de la Edad del Hierro se afirmaba, tradicionalmente, que no habían existido auténticas ciudades (Collis 1984; Wells 1984). En la actualidad sabemos que sí hubo núcleos que pudieron alcanzar más de un millar de habitantes (Guichard et al. 2000) e incluso asentamientos con superficies equiparables a las de los posteriores oppida, tal y como la investigación reciente ha demostrado en Heuneburg, de unas 100 Ha para la fase del Hallstatt D1 (Kurz 2005), y como parecen sugerir los trabajos en Bourges, donde varias "conurbaciones" ocupan unos centenares de hectáreas (Peyre y Buchsenschútz 2008) antes de la aparición de los primeros oppida. Por consiguiente, algunos autores consideran que los primeros centros urbanos al Norte de los Alpes se remontan a los siglos VI-V a.C. (Biel y Krausse 2005; Krausse 2005). La idea de que los oppida fueron las primeras ciudades europeas al Norte de los Alpes (Collis 1995; Wells 2002; Rieckhoff 2002) responde a la novedad que representan respecto a los asentamientos anteriores de la Edad del Bronce y de la Primera Edad del Hierro, pero choca con los criterios de "urbano" en otras civilizaciones (Herman Hansen 2000). Así Kolb (1984: 15) plantea seis criterios para definir una ciudad antigua: 1) desarrollo topográfico y administrativo, 2) más de 1000 habitantes, 3) diferenciación social y del trabajo, 4) instituciones ciudadanas, 5) morfología urbana y 6) función económica central. En todo caso, más que discutir con términos que no se definen claramente resulta mejor explicitar las características de los asentamientos de finales del Hierro y compararlos con sus precedentes y los de otras áreas culturales. Pues, como bien argumenta Collis (1996: 223), aunque "urbano" es un término útil para categorizar sociedades y permitir comparaciones culturales con sociedades de otras áreas y tiempos, nuestro objetivo no debe ser producir etiquetas o tipologías sino comprender cómo funcionaron y cambiaron las sociedades de la Edad del Hierro. Además, discutir si un sitio es urbano o no, es un ejercicio estéril en la medida en que muchas características de la categoría ciudad, como su estatus jurídicoideológico, no se encuentran en el registro arqueológico (Andreev 1989).

Aunque la demografía en los estudios arqueológicos ha sido, muchas veces, una especie de ciencia fantasma (Renfrew 2009), su valor es indudable, y eso a pesar de las serias dificultades para estimar densidades de población en la Prehistoria y que estos estudios hayan sido casi siempre periféricos en la investigación arqueológica (Bocquet-Appel 2008: 7). Es necesario vigorizar las aproximaciones que, desde el libro seminal de Hassan (1982), han permanecido relativamente adormecidas hasta hace pocos años (Bocquet-Appel 2008, Chamberlain 2006 y 2009) tras el impuso de los estudios de genética molecular. Contamos con estudios pioneros en la Edad del Hierro meseteña (Almagro-Gorbea 2001, Álvarez-Sanchís y Ruiz Zapatero 2001, Burillo 2005, Cerdeño y Sagardoy 2005-2006) y en la francesa (Isoardi 2009) que deben desarrollar metodologías y aplicaciones más sofisticadas. La demografía es un parámetro fundamental en la aparición de las ciudades y la extensión de sus territorios urbanos.

\section{El proceso de urbanización en la Edad del Hierro de la Meseta}

Si algo queda claro tras la lectura de los estudios que aquí se compilan sobre el urbanismo del primer milenio a.C. es, sin duda alguna, la idea de unidad en algunas cuestiones generales $\mathrm{y}$, al mismo tiempo, la diversidad de trayectorias en las diferentes regiones que constituyen la Meseta Norte española. Evolución en el tiempo y el espacio que, considerada en una perspectiva de tiempo largo - un milenio aproximadamente -, produce un efecto caleidoscópico, con situaciones cambiantes y diferenciadas en las maneras en que las comunidades meseteñas organizaron sus formas de poblamiento y explotación de los paisajes circundantes, sus formas de habitar la tierra.

Como todo resumen, éste obviamente está condenado a dejar fuera muchos aspectos y muchos detalles - y posiblemente incluso a sesgar las propias palabras e ideas de los au- 
tores - pero intentará ofrecer una mirada de conjunto a los resultados más fiables y a los problemas y lagunas de conocimiento esbozados. Como narrativa-resumen de las narrativas de los procesos de urbanización presentadas la voy a organizar de forma sintética y ciertamente muy procesual: como las visiones que, en diferentes tiempos, pudo tener un viajero protohistórico extraño a las tierras del interior peninsular, acaso viniendo desde el SO y subiendo por la Vía de la Plata, para luego a la altura del río Duero remontar su curso hasta las comarcas del Ebro medio en la "primera visita". Y en las posteriores miradas de imaginarios viajeros hasta el cambio de era, tal vez llegando desde las costas del Levante mediterráneo, penetrando por el Valle del Ebro y atravesando la Meseta, de Este a Oeste, desde el reborde del Sistema Ibérico hasta las sierras zamoranas y las estribaciones de los Montes de León. Las miradas a la situación del poblamiento meseteño serán las siguientes: la primera a la altura de alrededor del cambio de los siglos X-IX a.C., la segunda al filo del año 700 a.C., la tercera en el tránsito de los siglos V-IV a.C., la cuarta sobre el final del siglo III y los inicios del II a.C. y la quinta a finales de la primera centuria antes de la era.

\section{- Primera mirada (s. X-IX a.C.).}

Al entrar en la Meseta por la brecha del Jalón la primera impresión del imaginario viajero en este momento es la de un poblamiento no muy denso, disperso e integrado por comunidades pequeñas y muy pequeñas. Pocas columnas de humo levantándose a lo largo de los valles y de las campiñas - y todavía menos en los rebordes montañosos que cierran la cuenca del Duero -, delatando la existencia de pequeños aldeas con cabañas de materiales perecederos. En el centro de la cuenca los poblados y alquerías o granjas de Cogotas I acogen a un puñado de grupos familiares, probablemente nunca más de uno o como mucho dos centenares de habitantes, que ocupan espacios de menos de $2 \mathrm{Ha}$, con emplazamientos preferentes en llano, en ocasiones sin ningún tipo de defensa y en algunos casos más excepcionales con fosos que circundan el espacio doméstico y demuestran una inversión de trabajo y una vocación de, al menos, cierta permanencia.

Los grupos cogoteños practican una economía mixta, cultivan cereales, sobre todo trigo común como atestiguan los numerosos dientes líticos de hoz, algunos molinos de piedra, los silos - que no otra cosa parece que fueran la mayoría de los hoyos típicos de sus hábitats - y grandes recipientes de almacenaje. Los análisis de los territorios de explotación, como en el abulense Valle Amblés, indican una instalación preferente en fértiles suelos agrícolas. Poseen, además, rebaños de vacas, ovejas, cabras y algunos cerdos y caballos. Pero la movilidad es parte fundamental de su forma de vida, y aunque pueden permanecer varios años en los emplazamientos elegidos, junto a los lechos de inundación de los ríos o junto a orillas de pequeñas charcas endorreicas que salpican las campiñas, mueven sus poblados y granjas - probablemente volviendo repetidamente a los puntos previamente ocupados - lo que los convierte en "comunidades básicamente permanentes pero no por completo sedentarias", en palabras de los profesores de la universidad de Valladolid F. Romero y G. Delibes.

La situación descrita da cuenta del centro de la cuenca sedimentaria del Duero. En las tierras más al Occidente, en las serranías zamoranas y las estribaciones de los Montes de León, la presencia de estos hábitats de Cogotas I es casi imperceptible, quizás por estar escasamente pobladas en este periodo. Algo parecido sería el paisaje en las comarcas orientales: Alto Tajo/Alto Jalón y Alto Duero; aquí a la débil presencia de las gentes cogoteñas hay que añadir la existencia de pequeñas agrupaciones de cabañas - El Palomar en Soria y Fuente Estaca en Guadalajara - que por las cerámicas acanaladas que incluyen indican su origen en los grupos de Campos de Urnas del Ebro Medio y Bajo Aragón. Parecen grupos en movimiento que desde la línea del Ebro se adentran en las tierras altas del oriente de la Meseta. Aunque con algunas dudas sobre su cronología y adscripción cultural, los primeros indicios del ritual de cremación parecen estar en estas comarcas: la necrópolis de Herrería (Guadalajara) en las fases I y II y la soriana de San Pedro en Oncala. 


\section{- Segunda mirada (ca. 700 a.C.).}

Han transcurrido ocho o nueve generaciones desde que esas pequeñas comunidades de Cogotas I y grupos emparentados con los Campos de Urnas en el Oriente fueran avistadas por el viajero. Esas generaciones representan, sin duda, el periodo más oscuro de la historia del primer milenio a.C. en la región. El nuevo visitante encuentra ahora un paisaje bastante distinto: los asentamientos no son ya simples agrupaciones de cabañas, los hábitats ofrecen una arquitectura doméstica dura, en piedra y adobe, y una arquitectura defensiva con algunas murallas y fosos, aunque no habría que descartar que fueran en algunos casos obras para la contención de inundaciones. El asentamiento es permanente: las estratigrafías y superposiciones en los poblados son inequívocas. Las comunidades se han hecho visibles en el paisaje y sus efectivos también parecen haberse incrementado de forma paralela.

En el centro de la cuenca del Duero, sobre todo en los afluentes de la margen derecha, los poblados, con casas de planta circular construidas en adobe, forman verdaderos tells y ofrecen las "primeras trazas urbanas", en feliz expresión de Romero y Delibes. Es el Soto Inicial o Formativo (ca. 900-700 a.C.). Las ocupaciones más antiguas apenas ofrecen unos pocos solapamientos con sitios de Cogotas I y, aún en estos casos, no parece probada la continuidad de poblamiento. Lo que plantea la difícil pregunta de si las gentes del Soto son el resultado de una llegada de nuevas poblaciones provenientes del Este, del Valle del Ebro, o si por el contrario son simplemente la evolución y adaptación a nuevas circunstancias de las comunidades de Cogotas I. Nos falta información del "periodo oscuro" (s. X-IX a.C.) pero quizás la clave resida en una articulación, todavía no bien comprendida, de los siguientes componentes: a) una continuidad poblacional básica, además de b) nuevas situaciones climáticas y ambientales y c) un estímulo, cultural más que demográfico, llegado desde los grupos incineradores del área del Ebro.

Fuera como fuese, la realidad es que al filo de comienzos del siglo $\mathrm{X}$ a.C. comienzan a levantarse los poblados de tipo Soto. Las casas redondas, pequeñas - y algunas estructuras es- peciales con función de almacén y otras menos claras -, entroncan con la tradición circular de las cabañas cogoteñas pero su construcción en adobe es una completa innovación. Algo parecido se podría decir de la distribución de las casas sin orden aparente en los poblados soteños, por más que éstos sean de mayor superficie que sus predecesores de Cogotas I. Aunque esto último es posible que sea más bien fruto de la falta de excavaciones en extensión, ya que cuando éstas se realizan (La Guaya y La Corona/El Pesadero) se localizan calles y callejas, empedradas algunas, y agrupaciones de estructuras que podrían ser "unidades familiares de ocupación", lo cual constituye las primeras trazas de urbanismo en la región.

¿Pero de dónde procede la fuerza y pujanza de las gentes soteñas? El viajero que entrara en un poblado de alrededor del año 700 a.C. descubriría abundantes cantidades de cereal - con régimen de monocultivo y barbecho y posible rotación de cultivos - en las casas, auténtica riqueza de estas comunidades, y una activa ganadería con los bóvidos como primera cabaña en importancia, con ovejas y cabras en segundo lugar, algo de cerdo y algunos caballos. La agricultura cerealista extensiva y el vacuno eran la base de subsistencia. ¿Tuvo algo que ver esa orientación económica con las nuevas condiciones del inicio del periodo Subatlántico? Es muy posible que fuera así y, de hecho, creo que la investigación en esa dirección puede ser una de las más prometedoras en el inmediato futuro.

En el margen occidental zamorano los típicos poblados soteños parecen tener un protagonismo propio y formar parte del mundo del Soto más que constituir una deriva cultural. El poblamiento es disperso y poco jerarquizado, siguiendo los valles fluviales, con castros fortificados de pequeño tamaño, raramente más de $6 \mathrm{Ha}$, que albergarían comunidades de poco más de 100 habitantes y podrían llegar a lo sumo a tres o cuatro centenares. Pero en el extremo oriente meseteño el paisaje que encontraría el viajero resulta más pobre: comarcas muy poco pobladas, con apenas pequeños grupos suprafamiliares en cabañas que parecen ser avanzadillas pioneras de gentes del Ebro, como El Palomar (Almajano, Soria). Parecen representar el mismo fenómeno de enclaves 
algo anteriores del tipo de Fuente Estaca. Dos o tres generaciones más tarde de la visita del viajero - con los hijos y los nietos de aquellos que conoció - comenzará un movimiento activo de construcción de poblados en alto, muchos de nueva planta, con arquitectura de piedra y sus cementerios de incineración anejos. Por las tierras duras y frías del Alto Duero y las cabeceras del Tajo y el Jalón se multiplicarán los castros fortificados en altura y las necrópolis de incineración. Estímulos llegados del Ebro, y en última instancia de las costas mediterráneas, no debieron ser ajenos pero la clave de la génesis del mundo castreño, que razonablemente cabe ligar a la emergencia de los celtíberos, nos resulta todavía elusiva.

\section{- Tercera mirada (ca. 400 a.C.).}

La tierra ha recibido los restos de varias generaciones de soteños, sin que conozcamos su ritual funerario. Las decenas y decenas de aldeas soteñas dispersas han ido languideciendo en número y cuando nuestro viajero recorre el centro de la cubeta del Duero el paisaje ha cambiado sustancialmente. Ahora los núcleos de población se han reducido drásticamente y como fruto de un proceso de sinecismo apenas quedan algo más de medio centenar de asentamientos. Los grupos parentales bajo la dirección de líderes, cuya naturaleza desconocemos - y debería ser uno de los focos futuros de investigación - se concentran sobre todo en algunos de los antiguos poblados ya que se realizan pocas fundaciones nuevas y éstas configuran un modelo peculiar: el de los grandes oppida vacceos. Por primera vez surgen núcleos grandes (5-20 Ha, algunos de $25 \mathrm{Ha}$ y unos pocos de más de $40 \mathrm{Ha}$ ), distantes entre sí (10 y hasta $20 \mathrm{~km}$ ), con muy escasa jerarquización entre ellos y dotados de auténticas tramas urbanas y una alta densidad de ocupación en los sectores residenciales amurallados. El patrón de asentamiento contempla áreas de concentración de la población, especialmente en los valles de los ríos, junto a amplias comarcas prácticamente vacías, los famosos "vacíos vacceos" en ingeniosa expresión de J. D. Sacristán.

Para el viajero que se aproxima a una de estas ciudades vacceas, ya constituyéndose a comienzos de la cuarta centuria a.C., la contemplación resulta llamativa. La ciudad es muy grande, tanto que puede albergar de 1500 a 5000 habitantes, y los cientos de columnas de humo que se levantan de los tejados configuran una densa nube recortándose en el cielo. Las murallas y el foso que encierran todo el espacio urbano destacan todavía más a la ciudad. La vida bulle también fuera. La atenta mirada del viajero descubre a numerosos artesanos afanándose en alfares y otras instalaciones que se levantan fuera de las murallas; en otros puntos extramuros cuadrillas de hombres vacían carros con escombros de casas arruinadas y otros realizan vertidos de desechos domésticos. Más lejos, en una zona improductiva, el amplio espacio cementerial se reconoce por las estelas que marcan las tumbas de incineración y algunas figuras se mueven lentamente entre los enterramientos. Y todavía más allá se divisan los extensos campos de cereal, con superficies de cultivo nunca vistas antes - varios centenares de hectáreas - que constituyen la base de subsistencia, junto a los numerosos rebaños de vacas que aprovechan los rastrojos y algunas cabras y ovejas. Algunos jinetes se mueven junto a los bóvidos.

Al traspasar las puertas se abre una intrincada red de calles empedradas y con aceras que forman - siguiendo un modelo ortogonal - manzanas de casas rectangulares levantadas con adobe, con las traseras compartidas y abriendo sus puertas a una y otra calle, lo que implica una planificación inicial y el desarrollo de un plan unitario. Aparentemente no se ven edificios públicos diferenciados de los domésticos. Las casas, con plantas rectangulares divididas en tres espacios, nos resultan mal conocidas, en el sentido de que apenas contamos con alguna excavación que descubra en área plantas completas y la posibilidad de comparar los equipamientos domésticos.

Este modelo, que J. D. Sacristán ha denominado expresivamente "ciudad-estado uninuclear vaccea", porque las ciudades controlan territorios modulares de $400 / 500 \mathrm{~km}^{2}$ en los que apenas existen otros núcleos menores, se va forjando a lo largo del s. IV a.C. y en su opinión está ya constituido, al menos en sus rasgos esenciales, en los albores del s. III a.C. Este modelo presenta el reto de precisar los factores 
que conducen al proceso de sinecismo comentado. Parece indudable una intensificación de la producción en la que, acaso, el instrumental de hierro que ahora se generaliza - y muy especialmente el arado - jugó un papel de primer orden y estuvo en la base de los cambios que se operaron. La adaptación a una economía agraria fundamentalmente cerealista extensiva de secano, y la vertebración de los grupos parentales en una nueva organización social con elites, que rastreamos en las tumbas más ricas de los pocos cementerios vacceos conocidos como Las Ruedas de Padilla de Duero, Palenzuela o Cuéllar, pudieron ser las claves del exitoso modelo vacceo que se mantendrá por muchas generaciones.

Al SO de la línea del Duero, traspasadas las campiñas, el viajero entra en tierras de vettones. Tierras de granito, pastos y encinares en las que se asiste ahora al surgimiento de los oppida, que agrupan las poblaciones de las pequeñas aldeas de la etapa anterior. La mayoría de los asentamientos del s. IV a.C. son de nueva planta. El poblamiento se concentra en valles fértiles y protegidos, como los de Amblés, Yeltes y Huebra, y deja grandes áreas casi vacías. Los centros vettones - Las Cogotas, La Mesa de Miranda, El Raso, Yecla de Yeltes - tienen murallas de piedra, fosos y bandas de piedras hincadas en los accesos más fáciles, que dan empaque a sus pobladores, no en balde las defensas adquieren también un valor simbólico de exhibición de la riqueza y pujanza de las comunidades que viven en ellos. Las ciudades vettonas encierran grandes superficies (de 10 a más de $30 \mathrm{Ha}$ y excepcionalmente Ulaca, con algo más de $70 \mathrm{Ha}$ ). Sus interiores se compartimentan a veces con varios recintos cerrados $\mathrm{y}$, a los ojos del viajero, ofrecen un aspecto muy distinto a las ciudades vacceas; aquí las casas de planta rectangular o cuadrangular con departamentos internos son insulanas, no siguen ningún ordenamiento urbanístico más allá de acomodarse a las topografías y especialmente las vaguadas que las atraviesan. Casas aisladas dispersas, bajas para defenderse del frío y con cubiertas vegetales de piorno y otros arbustos, que dejan muchos espacios libres con una baja densidad de ocupación. Salvo el santuario rupestre y una sauna iniciática excavada también en la roca de Ulaca, no se advierten edificios públicos. Los efectivos de población son menores que en la zona vaccea por la débil ocupación con caserío disperso. Debieron contarse entre dos o tres centenares de habitantes y el medio millar; en los casos más grandes superarían en poco los 1000 -1500 habitantes.

La importancia de la ganadería ayuda a comprender el valor de las esculturas de toros y cerdos, los famosos "verracos", como elementos usados por las elites de los oppida para reclamar la propiedad y el derecho de uso de pastos críticos en las épocas de calor y estiaje elevado. Así en los pastizales alrededor de los oppida menudean las esculturas de granito, auténticos hitos demarcadores de su explotación. Los cementerios, con túmulos pétreos y tumbas de incineración en hoyo, al lado de los oppida, complementan la inserción de las comunidades vettonas en el paisaje.

En el camino hacia el oriente meseteño el viajero se encuentra con pequeños poblados en alto y en llano en las tierras bajas del Alto Duero, mientras que en la serranía soriana los castros de la fase anterior han crecido en número y también en tamaño, aunque en cualquier caso son mayoría los pertenecientes a muy pequeñas comunidades (menos de $1 \mathrm{Ha}$ ); incluso los mayores apenas rebasan las 2 Ha. (Fig. 1). Pequeñas aldeas serranas autosuficientes, con murallas de piedra, que salpican alturas entre los 900 y poco más de $1200 \mathrm{~m}$. Al Sur de Soria capital los llanos de cereal no tienen todavía centros que merezcan el nombre de ciudades, y eso que a pocos kilómetros al Este, bajando por los afluentes del Ebro, en las tierras medias de este gran valle ya existían ciudades celtíberas en el s. V a.C. Todavía transcurrirán cinco o seis generaciones hasta que en el Alto Duero aparezcan las primeras ciudades. Y así Numancia y Tiermes, con indicios de ocupación en el momento de la travesía de nuestro viajero, no tienen nada de las grandes ciudades celtíberas que más adelante llegarán a ser.

En las cabeceras del Tajo y del Jalón los castros de la fase anterior se encuentran pujantes, crece la población y se advierte también una intensificación económica que la sostiene. Algunos centros como El Ceremeño sufren importantes reordenaciones internas y por todos lados parecen incrementarse las defensas de los castros con murallas, fosos y campos de 


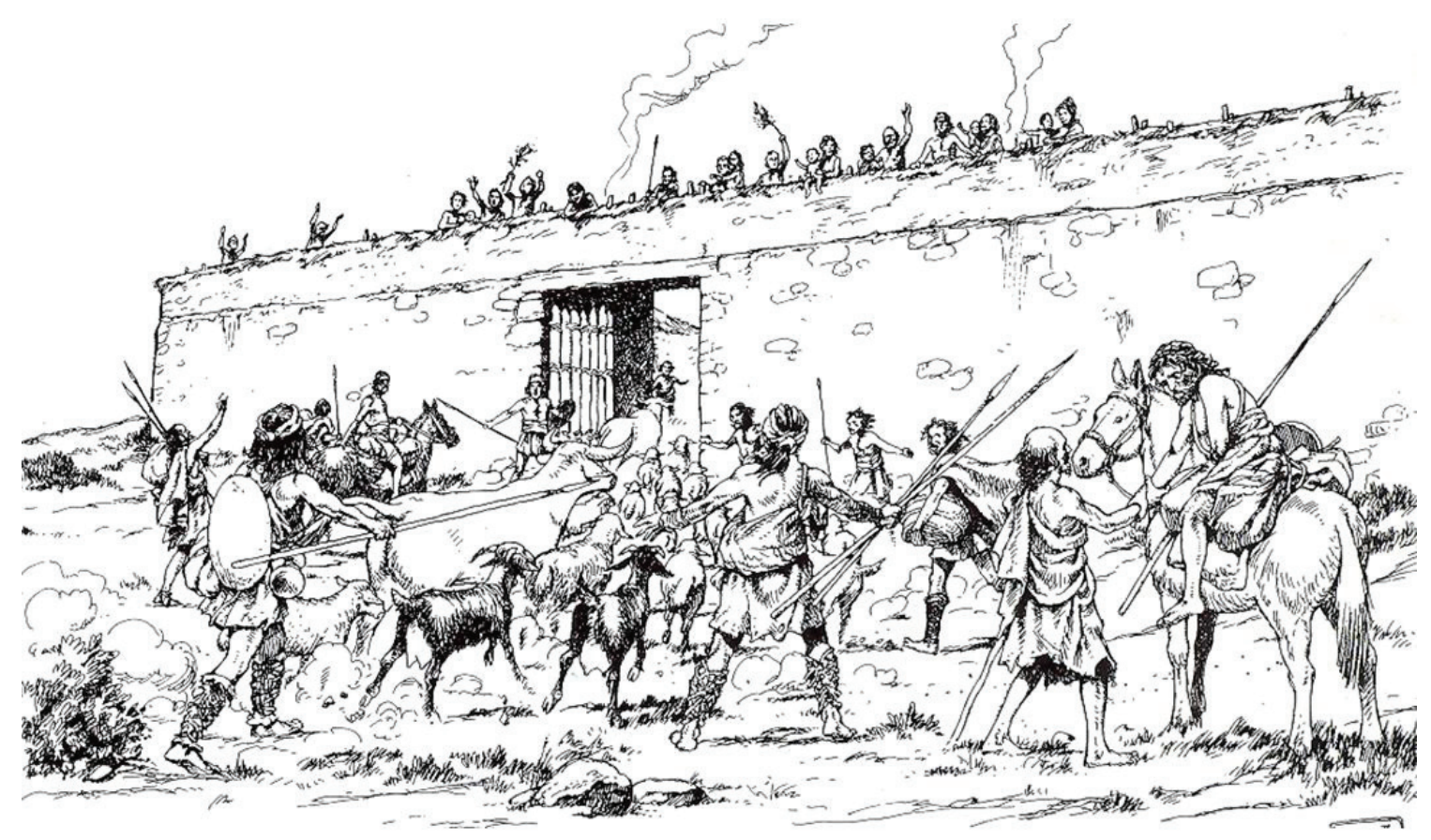

Figura 1.- Recreación de una comunidad castreña del Oriente de la Meseta (Benito et al. 2006).

piedras hincadas (Peña Moñuz, El Hocincavero, Torre de Codes). En todo caso el aspecto de estos poblados recuerda más los típicos castros de la fase inicial que lo que serán las ciudades celtibéricas. La demografía de los mismos corrobora, de alguna manera, esta afirmación.

\section{- Cuarta mirada (ca. 200 a.C.)}

Con el horizonte de las primeras incursiones romanas en las tierras de la Meseta las viejas tradiciones de los distintos populi cristalizan en el mundo urbano que, ahora ya sí, se va generalizando por todas las regiones y comarcas. El viajero de este momento encuentra continuidades y novedades pero las comunidades meseteñas van dejando atrás elementos de sus raíces originarias. Continuidades son, por ejemplo, las que presenta el mundo vacceo en el Duero Medio. Las grandes ciudades vacceas están iniciando su apogeo, caso de Montealegre y Pintia, en Padilla de Duero, un gran centro urbano que pudo albergar 6000 ó 7000 habitantes, con cementerios, áreas industriales y artesanales, un santuario y arrabales extramuros (Fig. 2). Ciudades que sin duda desarrollaron en su dimensión política senados, magistraturas y jefaturas militares, y que controlaron y explotaron los dominios del campo en territorios de muchos centenares de $\mathrm{km}^{2}$. Cuestiones éstas que precisan más investigación en los cementerios, para poder acercarnos a su estructura y organización social $\mathrm{y}$, sobre todo, en las propias anatomías internas de las ciudades, algo que sólo muy escasamente estamos empezando a vislumbrar.

En la cabecera del Duero, el viajero contempla ahora las grandes ciudades celtíberas que iniciaron su andadura a finales del siglo III a.C.: Numancia, Uxama, Tiermes, Arekoratas (Muro de Agreda), Segontia Lanca, Clunia y Contrebia Leucade, por citar sólo las más grandes y famosas. El aspecto de estas ciudades celtibéricas, protegidas con murallas, es de un urbanismo ortogonal con sistemas de calles que se cruzan, en un modelo que parece simplemente la ampliación del esquema antiguo de los "poblados de espacio central", sin arquitectura monumental pública y con una 


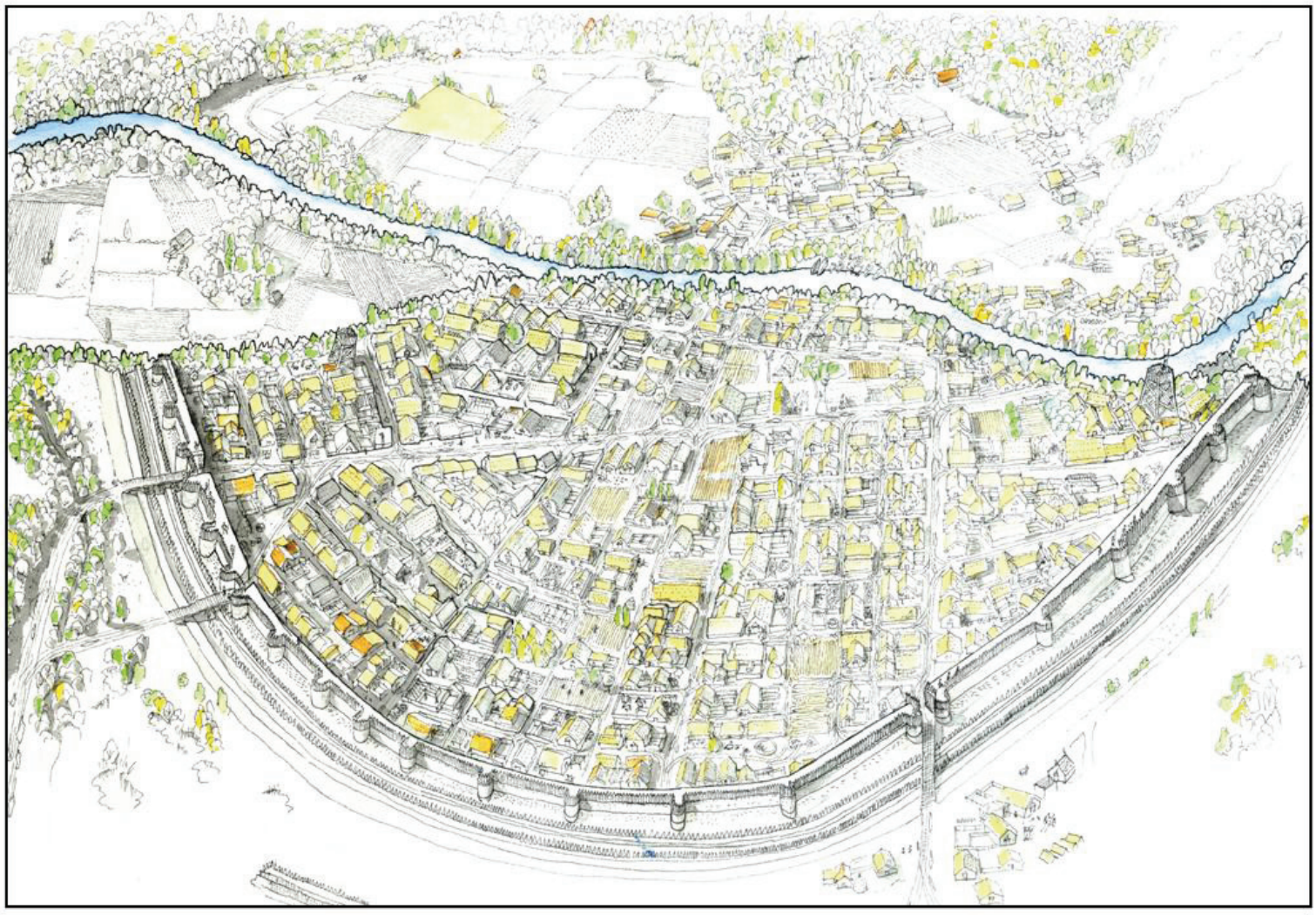

Figura 2.- Reconstrucción ideal de la ciudad de Pintia (Según P. Sainz Guerra - El Norte de Castilla; cortesía del Centro de Estudios Vacceos "Federico Wattenberg" de la Universidad de Valladolid). Aún cuando algunos detalles de la imagen son muy conjeturables, dada la limitada excavación en área de la trama urbana y de los sistemas defensivos, la idea que expresa de una gran ciudad vaccea sí resulta válida.

ocupación densa del espacio intramuros, ya que las áreas residenciales ocupan bastante más superficie que los ámbitos públicos. Esto último se traduce en una alta densidad de poblamiento (208 hab $/ \mathrm{km}^{2}$ en Numancia). Las casas, de planta rectangular y división interna tripartita, se adosan formando auténticas manzanas. La población absoluta no es fácil de calcular pero para la Numancia de esta época, con poco más de $4 \mathrm{Ha}$ de extensión, se ha estimado una población de unos 1500 habitantes. Las ciudades celtíberas eran ciudades-estado autónomas que estaban dotadas de consejos, magistrados y asambleas y jefes militares nombrados en periodos de conflicto, y que sólo se entendían en relación con el campo que controlaban. De hecho, el modelo celtibérico se caracteriza por una intrincada red de aldeas fortificadas y entidades rurales menores que acogen a la mayoría de la población y también a las aristocracias urbanas. Como dice F. Burillo, no existía oposición campo/ciudad.

En el área occidental vettona, a comienzos del siglo II a.C., el modelo de los oppida asiste a una intensificación de la producción y una jerarquización del territorio. El viajero que atraviesa la región encuentra los centros fortificados, los grandes cementerios en uso y la erección de verracos que siguen marcando los pastizales. Las comunidades vettonas siguen siendo comunidades fuertes pero pocas generaciones más tarde, con la progresiva incorporación de los territorios a Roma, la autonomía y el esplendor de los grandes oppida entrará en declive. Y cuatro generaciones después, ya en la segunda mitad del s. I a.C., el sistema indígena empezará a disgregarse. El tiempo de los oppida se estaba acabando. Otro mundo empezaba a configurarse. 


\section{- Quinta mirada (finales del s. I a.C.): Epílogo}

En las postrimerías del siglo I a.C. las ciudades, poblados y castros que mantenían las poblaciones prerromanas experimentan - de forma desigual también -, el sometimiento al poder de Roma. Los sistemas de poblamiento autóctonos se van rápidamente transformando y la realidad indígena se va diluyendo en el proceso. La mirada del viajero descubre nuevos elementos que configuran las ciudades y la aparición de nuevas vías de comunicación con la red de calzadas romanas que alteran profundamente los paisajes y la percepción de la vida cotidiana. El mundo prerromano ha sido transformado profundamente. Los centros urbanos, el campo, los caminos y los paisajes, al cabo de un milenio son muy distintos a los de finales de la Edad del Bronce.

\section{Perspectivas de futuro y necesidades de la investigación}

La historia del urbanismo prerromano es la historia de la organización compleja del territorio, una ordenación sociopolítica del paisaje como bien señala Álvarez-Sanchís (este volumen). Una ordenación del paisaje que de las pequeñas comunidades autárquicas de comienzos del primer milenio a.C. terminará con una diversidad de paisajes, ciudades y otros tipos de asentamiento que acabarán disgregándose con el proceso de conquista romana (Fig. 3). Pero, al mismo tiempo, la historia del urbanismo prerromano es la historia del desarrollo de las sociedades campesinas simples de finales de la Edad del Bronce que, a través de diferentes fórmulas, devendrán en ciudades-estado en las postrimerías de la Edad del Hierro.

El urbanismo prerromano, más allá de la caracterización interna de los asentamientos, materializa, por un lado, la utilización del paisaje por parte de las comunidades del Hierro, expresando su categorización; y por otro, encierra, de alguna manera, la estructura social y la organización política de las gentes del primer milenio a.C.; en otras palabras: la urbanización es un fenómeno más de la historia social (Andreev 1989).

De las síntesis reunidas en este volumen cabe deducir una serie de problemas y limitaciones que reducen la visibilidad y comprensión de todas las dimensiones del urbanismo meseteño en la Edad del Hierro:

1) Una impresión global es la limitación de los proyectos y excavaciones arqueológicas en los yacimientos de la Edad del Hierro en la Meseta española. A pesar de la continuada tarea excavadora, y en menor medida prospectora, no hay grandes proyectos interinstitucionales del estilo de Alemania, como el Proyecto Frühe Zentralisierungs- und Urbanisierungsprozesse. Zur Genese und Entwicklung frühkeltischer Fürstensitze und ihres territorialen Umlandes (www.fuerstensitze.de), desarrollado por la $\mathrm{Co}$ munidad Alemana de Investigaciones (DFG) y financiado con unos 9 millones de euros (Biel y Krausse 2005); o los proyectos del Reino Unido sobre los paisajes de los hillforts de la Edad del Hierro (http://www.hillfortsstudygroup.org. $\mathrm{uk} / \mathrm{hsgbiblioengland.html).} \mathrm{Entre} \mathrm{nosotros,} \mathrm{en}$ los últimos veinticinco años de "arqueología autonómica" (de las Comunidades Autónomas) el desarrollo creciente de la arqueología de empresa - arqueología de gestión - ha ido acompañado de un cierto declive de la investigación arqueológica académica. Sin duda se ha excavado más que nunca, pero lo publicado representa una mínima parte. Y aún así hay que reconocer que muchas novedades vienen de los trabajos de las empresas de arqueología. No hay proyectos ambiciosos de largo plazo y no hay proyectos interinstitucionales, al tiempo que los resultados dispersos de la arqueología de gestión no se integran en proyectos unificados, como ya he señalado en más de una ocasión (Ruiz Zapatero 2007). Y tampoco participamos, o lo hacemos muy limitadamente a titulo personal, en redes temáticas europeas sobre la Edad del Hierro. Un punto de partida como el británico Understanding the British Iron Age (Haselgrove et al. 2001), una especie de libro blanco sobre la investigación de la Edad del Hierro, resultaría enormemente provechoso en el contexto español.

2) La variable paleoambiental parece muy importante para entender procesos de inicios de la Edad del Hierro relacionados con el comienzo del periodo Subatlántico (ca. 800 a.C.), pero la información disponible a nivel regional resulta bastante pobre. Hacen falta más datos paleoambientales contextualiza- 


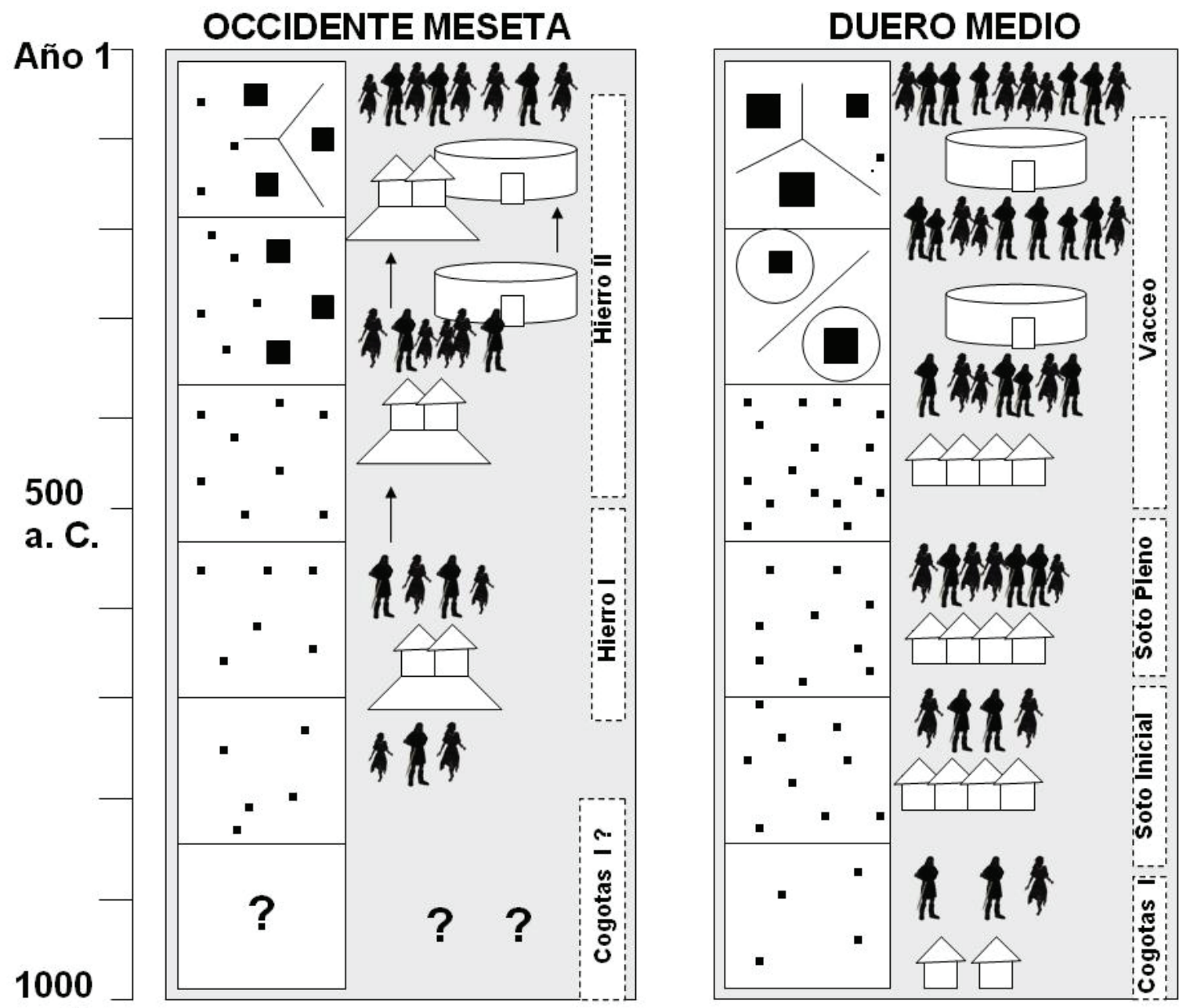

a. C.

Figura 3.- Diagrama evolutivo de los patrones de poblamiento, tipos de asentamiento, estimaciones demográficas y grupos arqueológicos en la Meseta Norte durante el primer milenio a. C.

dos por áreas que permitan ir más allá de las generalizaciones excesivas que no tienen en cuenta los detalles locales. Los cambios climáticos, de vegetación y fauna, pueden ayudar a precisar mucho mejor los procesos económicos y de subsistencia puestos en marcha por las comunidades de la Edad del Hierro meseteñas, que tuvieron consecuencias decisivas en la aparición y desarrollo del urbanismo.

3) Los datos de los Inventarios Arqueológicos Provinciales de la Junta de Castilla y León deben permitir explotar más la información disponible, así como las memorias sin publicar depositadas en las Administraciones Autonómicas. Y sin duda sería muy importante, de cara al estudio del urbanismo prerromano, la potenciación de los proyectos de prospección arqueológica. Especialmente en aquellas áreas y comarcas con pocos datos, auténticos "agujeros negros" en el paisaje protohistórico. Sólo así se podrá avanzar en la realización de estudios comparativos entre áreas con la base documental necesaria. Y aquí es donde hace falta más cooperación entre administraciones, universidades y empresas de arqueología. Y, además, cooperación 
ALTO DUERO

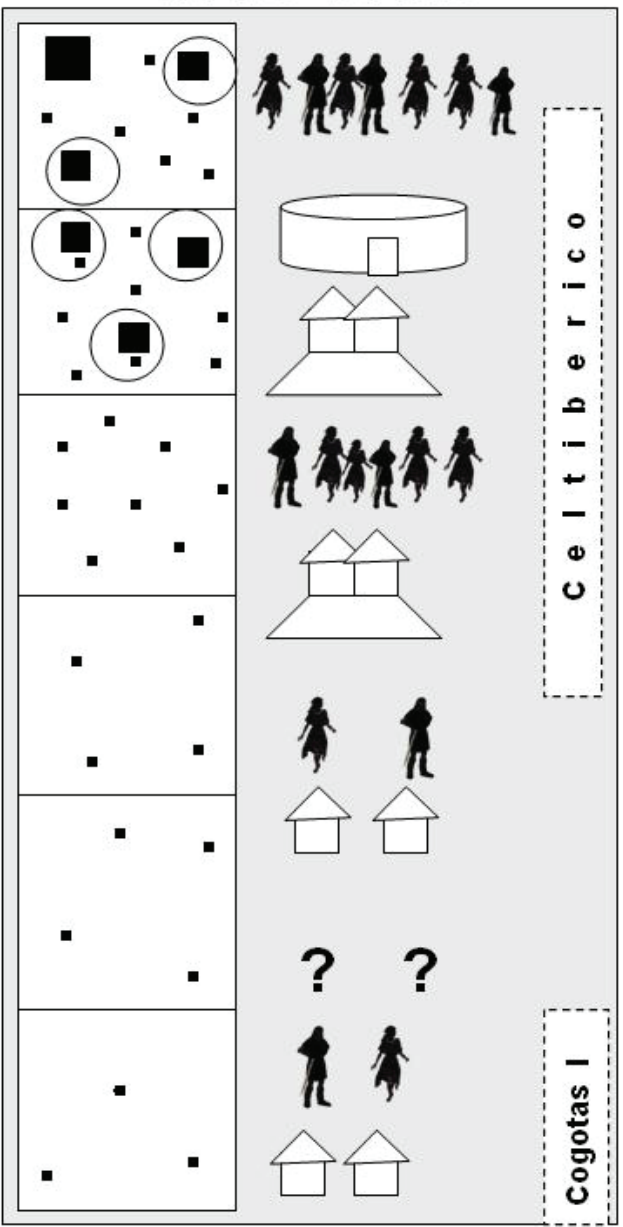

ALTO TAJO / ALTO JALÓN

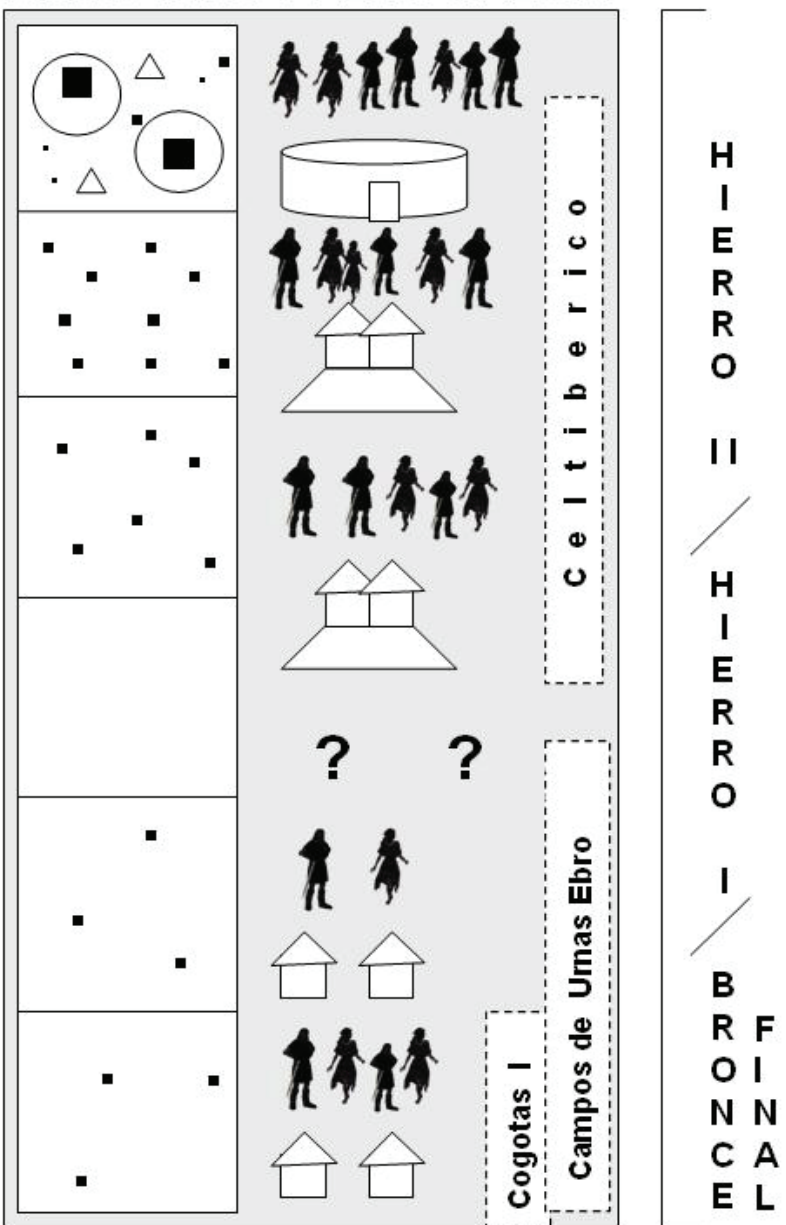

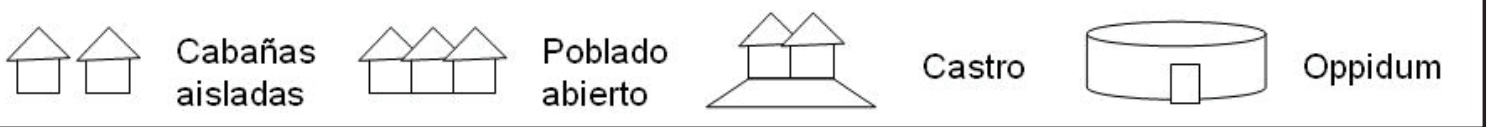

imaginativa que abra nuevos cauces y vías para aprovechar la ingente documentación que cada año se va generando. Algún trabajo pionero en este sentido (Blanco 2010) demuestra el valor de la propuesta y la potencialidad de los Inventarios de cara a los estudios de poblamiento y continuidad/discontinuidad de las ocupaciones de los distintos periodos.

4) Los estudios sobre la Edad del Hierro, sean de urbanismo o cualquier otro aspecto, deberían tener una perspectiva más europea. Los desarrollos del primer milenio a.C. en la Meseta se verían más enriquecidos si existiese una mayor preocupación por insertar sus interpretaciones en los desarrollos de otras regiones europeas con trayectorias que, a veces, son parecidas y en otras ocasiones diferentes. No estoy del todo seguro de si debe existir algo así como una "arqueología europea" (Kristiansen 2008), pero desde luego sí debe haber marcos europeos para el estudio de las sociedades de la Edad del Hierro. Visiones más panorámicas ofrecen, sin duda alguna, visiones más críticas. Y ciertamente el origen del urbanismo es un tema de claro alcance y valor europeo en el que grandes regiones supranacionales compartieron trayectorias, o al menos sus trayectorias tuvieron similitudes o simplemente interactuaron con resultados diversos: unidad y diversidad que 
caracterizan a la Edad del Hierro europea (Thurston 2009: 22-26). No deja de ser una paradoja, pero refleja muy bien lo señalado más arriba, que la única publicación sobre las sociedades complejas y la aparición del urbanismo en la Prehistoria final peninsular sean las actas de una reunión organizada en el Reino Unido (Cunliffe y Keay 1995), con una amplísima participación española invitada por los organizadores. Desde esa perspectiva el marco de Europa y sus regiones también me parece un mejor marco de referencia y menos excluyente que los dominantes - aunque ciertamente inevitables - marcos locales y nacionales (Kristiansen 2008: 25). Por otra parte, el predominio del paradigma histórico-cultural y la muy tardía y débil presencia de los enfoques procesuales, postprocesuales y materialistas-históricos, ayudan a entender la falta de estudios con una fuerte perspectiva comparativa en la Edad del Hierro española.
5) Es necesario desarrollar un mayor cuerpo teórico y metodológico sobre urbanismo desde la perspectiva arqueológica y para ello hay que tener una amplia base comparativa sobre las ciudades y el urbanismo en todo tiempo y espacio; es más, incluso se señala que para ello resulta crucial una mayor interacción entre arqueólogos y otros estudiosos del urbanismo, tanto histórico como contemporáneo (Smith 2010). Y desde luego cualquier intento de comprensión sistemática del urbanismo, histórica y comparativamente, debe incluir las ciudades antiguas (Smith 2009). Tres actuaciones parecen relevantes en este sentido: primero, la investigación del urbanismo antiguo empleando conceptos y categorías de los modernos estudios de urbanismo; segundo, la implicación de arqueólogos en proyectos de investigación transdisciplinares junto a otros especialistas, y tercero, la publicación de los estudios arqueológicos en revistas de fuera de la disciplina para difundir mejor y estimular la interacción (Smith 2010: 246).

\section{REFERENCIAS BIBLIOGRÁFICAS}

Almagro-Gorbea, M. (2001): Aproximaciones a la demografía de la Celtiberia. Entre Celtas e Iberos. Las poblaciones protohistóricas de las Galias e Hispania (L. Berrocal-Rangel, Ph. Gardes, eds.), Bibliotheca Archaeologica Hispana, 8, Real Academia de la Historia, Madrid: 45-60.

Álvarez-Sanchís, J. R.; Ruiz Zapatero, G. (2001): Cementerios y asentamientos: bases para una demografía arqueológica de la Meseta en la Edad del Hierro. Entre Celtas e Iberos. Las poblaciones protohistóricas de las Galias e Hispania (L. Berrocal-Rangel, Ph. Gardes, eds.), Bibliotheca Archaeologica Hispana, 8, Real Academia de la Historia, Madrid: 61-75.

Andreev, Y. V. (1989): Urbanization as a phenomenon of social history. Oxford Journal of Archaeology, 8 (2): $167-77$.

Benito, J. P., Tabernero, C., Sanz, A.; Guillen, R. (2006): Guía arqueológica. Pelendones. Castros célticos en la serranía norte de Soria. Editorial Areco, Soria.

Biel, J. y Krausse, D. (eds.) (2005): Frühkeltische Fürstensitze. Älteste Städte und Herrschaftszentren nördlich der Alpen? Internationaler Workshop zur keltischen Archäologie in Eberdingen-Hochdorf 12. und 13. September 2003. Archäologische Informationen aus Baden-Württemberg 51, Esslingen.

Blanco GonzÁlez, A. (2010): ¿Nuevos hogares para los emigrantes? Casas y paisajes en el debate sobre el límite entre Cogotas I y el Primer Hierro en el Valle del Duero. Zephyrus, LXVI (julio-diciembre):155-179.

Bocquet-APPel, J.-P. (2008): La Paléodémographie. 99,99\% de l'histoire démographique des hommes ou la démographie de la Préhistoire. Éditions Errance, Paris.

Burillo, F. (2005): Aproximación a la demografía de la ciudad celtibérica de Segeda I. Mayurqa, 30: 827-847.

Cerdeño, M.L., Sagardoy, T. (2005-2006): Estudio demográfico sobre el Celtibérico Antiguo. Kalathos, 24-25: 219-237.

Chamberlain, A.T. (2006): Demography in Archaeology. C. U.P., Cambridge. 
Chamberlain, A.T. (2009): Archaeological Demography. Human Biology, 81 (2-3): 275-86.

Coluis, J., (1984): Oppida: Earliest Towns North of the Alps. University of Sheffield, Sheffield.

Coluis, J. (1995): The first towns. The Celtic World (M. A. Green, ed.), Routledge, Londres: 159-175.

Collis, J. (1996): Urbanisation in Atlantic Europe in the Iron Age. Gallaecia, 14-15: 223-241.

Cunliffe, B.; Keay, S. (eds.) (1995): Social Complexity and the Development of Towns in Iberia: From the Copper Age to the Second Century A.D. Oxford University Press, Oxford.

Guichard, V.; Sievers, S.; Urban, O.H. (eds.) (2000): Les processus d'urbanisation à l'Age du Fer. Eisenzeitliche Urbanisationprozesse. Colloque 8-11 juin 1998. Centre Archéologique Européen du Mont Beauvray, Glux-en-Glenne,

Haselgrove, C.; Champion, T. C.; Armit, I.; Creighton; J.; Gwilt, A. (2001): Understanding the British Iron Age: An Agenda for Action. A report for the Iron Age Research Seminar and the Council of the Prehistoric Society. Trust for Wessex Archaeology Ltd., Salisbury. (http://www.personal.reading.ac.uk/ lascretn/IAAgenda.htm).

Hassan, F. (1982): Demographic Archaeology. Academic Press, Nueva York.

Herman Hansen, M. ed. (2000): A Comparative Study of Thirty-City State Cultures. An investigation conducted by the Copenhaguen Polis Centre. The Royal Danish Academy of Sciences and Lettres, Copenhague.

IsOARDI, D. (2009): L'apport des donnés démographiques dans une étude de l'organisation de la société préromaine de France méridionale. Une nouvelle lecture des oppida. L'espai domèstic i l'organització de la protohistoria de la mediterrània occidental (1r mil.lenni aC) (C. Belarte, ed.), Actes de la IV Reunió Internacional d'Arqueologia de Calafell: 67-77.

Kolb, F. (1984): Die Stadt im Altertum. Beck, Munich.

KRAuSSE, D. (2005): Vetternwirtschaft? Fragestellung und Design eines archäologisch-paläogenetischen Pilotprojekts zur sozialhistorischen Deutung späthallstattzeitlicher Elitegräber? Frühkeltische Fürstensitze. Älteste Städte und Herrschaftszentren nördlich der Alpen? (J. Biel, D. Krausse, eds.), Internationaler Workshop zur keltischen Archäologie in Eberdingen-Hochdorf 12. und 13. September 2003. Archäologische Informationen aus Baden-Württemberg 51, Esslingen: 63-66.

Kristiansen, K. (2008): Do we need "the archaeology of Europe"? Archaeological Dialogues, 15 (1): 5-25.

KuRz, S. (2005): Zentralort und Umland: Untersuchungen zur Struktur der Heuneburg-Außensiedlung und zum Verhältnis der Heuneburg zu den umgebenden Höhensiedlungen. Resumée. (http://tobias-lib.uni-tuebingen.de/volltexte/2005/2076/pdf/Aussensiedlung).

Peyre, Сh. y Buschsenschütz, O. (2008): Tite-Live, Bourges, et les premiers processus d'urbanisation à l'âge de Fer en France septentrional. Germania, 86: 29-46.

Renfrew, C. (2009): Demography and Archaeology. Human Biology, 81 (2-3): 381-84.

RiecKHOFF, S. (2002): Der Untergang der Städte. Der Zussammenbruch des keltischen Wirtschafts- und Gesselschaftssystems. Dürnberg und Manching. Wirtchshaftsarchäologie im ostkelischen Raum (C. Dobiat, S. Sievers, Th. Stöllner, eds.), Bonn, Habelt: 359-379.

Ruiz Zapatero, G. (2007): Antes del Hierro. Cultura y sociedad en el centro de la Meseta (ca. 1200-500 a.C.). Estudios sobre la Edad del Hierro en la Carpetania. (A. Dávila, ed.), Zona Arqueológica 10, vol I, Museo Arqueológico Regional, Alcalá de Henares: 36-62.

Sмiтн, M. E. (2009): Editorial: Just how comparative is comparative urban geography?: A perspective from archaeology, Urban Geography, 30: 113-117.

Sмiтн, M. E. (2010): Sprawl, Squatters and Sustainable Cities: Can Archaeological Data Shed Light on Modern Urban Issues? Cambridge Archaeological Journal, 20 (2): 229-253.

Thurston, T. L. (2009): Unity and diversity in the European Iron Age: out of the mists, some clarity? Journal of Archaeological Research, 17(4): 7-84.

Wells, P. S. (1984): Farms, Villages and Cities. Commerce and urban origins in Late Prehistoric Europe. Cornell University Press, Ithaca.

Wells, P.S. (2002): The Iron Age. European Prehistory. A survey (S. Milisauskas, ed.), Kluwer Academic, Plenum Publishers, Nueva York - Londres: 335-384. 